\title{
INDICADORES
}

\section{RANKING ESCO DE AGENCIAS DE PUBLICIDAD: DESCRIPCIÓN Y RESULTADOS}

\author{
Rafael Repiso, Carmen Llorente-Barroso y Francisco García-García
}

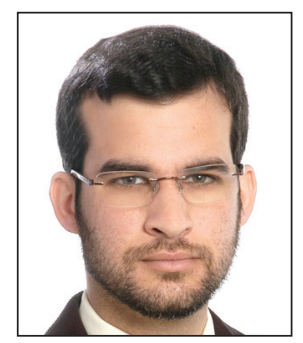

Rafael Repiso es diplomado en biblioteconomía, licenciado en documentación y máster en evaluación científica. Miembro del grupo de investigación EC3, es profesor en la ESCO, Escuela Superior de Comunicación de Granada, centro adscrito a la Universidad de Gales. Coeditor de las revistas Comunicar e Icono14. Co-fundador de www.sexenios.com. http://orcid.org/0000-0002-2803-7505

ESCO - Escuela Superior de Comunicación, Biblioteca Central San Antón 73. 18006 Granada, España rafael.repiso@gmail.com

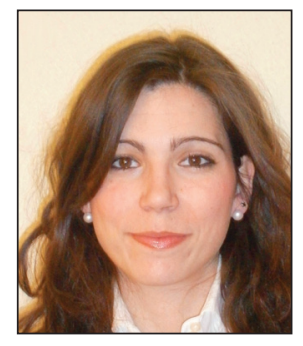

Carmen Llorente-Barroso, doctora en publicidad y comunicación audiovisual por la Universidad Complutense de Madrid (UCM), ha desempeñado parte de su carrera como investigadora OTRI UCM-Zenithoptimedia con cargo a un proyecto de eficacia publicitaria. Miembro activo de R14, CEU-Citec y Asocrea, compagina sus labores docentes en la Universidad CEU San Pablo con las investigadoras.

http://orcid.org/0000-0001-7710-0956

Univ. CEU San Pablo, Fac. de Humanidades y Ciencias de la Comunicación Po de Juan XXIII, 6. 28040 Madrid, España carmenllore@gmail.com

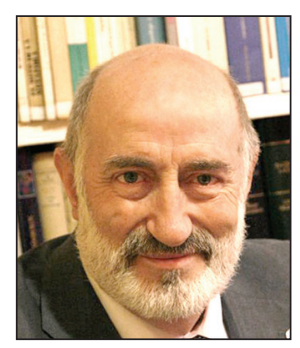

Francisco García-García, licenciado y doctor en ciencias de la información, es catedrático de comunicación audiovisual y publicidad en la Facultad de Ciencias de la Información de la Universidad Complutense de Madrid. Es director del grupo de investigación Icono 14 y de la revista del mismo nombre.Miembro desde 2000 de la Academia de las Ciencias y las Artes de Televisión.

http://orcid.org/0000-0001-5394-4804

Universidad Complutense de Madrid Facultad de Ciencias de la Información Ciudad Universitaria. 28040 Madrid, España fghenche@gmail.com

\section{Resumen}

Se presenta y describe el Ranking ESCO de agencias de publicidad, meta-producto que evalúa la calidad de las agencias a través de sus productos creativos y según una ponderación rigurosa de los premios que obtienen y los festivales en los que son galardonados. Se ha seleccionado una muestra de festivales clasificados en 4 categorías según su importancia nacional e internacional, considerando un período de carga de dos años para garantizar que entre cada edición exista un elemento de continuidad y otro de novedad. Este ranking pretende subsanar una manifiesta carencia en España respecto a otros países, al ofrecer no sólo una clasificación de las mejores empresas y trabajos publicitarios, sino también un análisis de la industria publicitaria española. Los resultados del ranking para 2012 registran 197 agencias, 611 anuncios y 359 anunciantes.

\section{Palabras clave}

Ranking, Agencias de publicidad, Ranking de publicidad, Premios de publicidad, Diseño de rankings.

Título: ESCO ranking of advertising agencies: description and results

\section{Abstract}

This paper presents and describes the ESCO Ranking of advertising agencies. The objective of this tool is to assess the quality of the agencies, based on their creative products and a rigorous evaluation of the various prizes received and the prestige of the festivals that granted them. For that purpose, festivals were classified into 4 categories based on their national and 
international importance and a sample was selected. A two-year period was considered for the ranking to ensure a balance of continuity and of innovation in each edition. This work aims at covering a gap in Spain as compared with other countries by providing not only a classification of the best advertising agencies but also an analysis of the advertising industry in Spain. The results of the 2012 edition include 197 agencies, 611 advertisements and 359 advertisers.

\section{Keywords}

Advertising agencies, Advertising ranking, Advertising award shows, Design rankings.

Repiso, Rafael; Llorente-Barroso, Carmen; García-García, Francisco (2013). “Ranking ESCO de agencias de publicidad: descripción y resultados". El profesional de la información, marzo-abril, v. 22, n. 2, pp. 181-187.

\section{Introducción}

Ocupar posiciones en el top de los rankings de publicidad es una de las prioridades de las agencias, especialmente de los grupos multinacionales. La implantación de rankings de publicidad comienza a principios de los sesenta en Norteamérica (Kübler, 2012). Desde entonces han surgido diferentes tipos que miden variables como los ingresos de las empresas, la calidad percibida a través de encuestas a expertos o los premios cosechados en diferentes festivales. En un principio se impusieron los que tomaban como principal variable de análisis diversos aspectos económicos. Sin embargo, cuando en 2002 se publica el Acta Sabannes-Oxley, las empresas estadounidenses dejan de publicar sus datos de facturación y la falta de información fiable sobre sus cuentas lleva a considerar otras variables de análisis en la ponderación de los rankings, especialmente la creatividad (Tippins; Kunkel, 2006). Años antes Helgesen (1994) definía la creatividad como la característica más admirada en el sector publicitario y probablemente el criterio más importante para determinar el éxito de una agencia. Las agencias que quieren participar en estos sistemas de evaluación acuden a festivales y premios donde la creatividad es especialmente valorada, con el objetivo de ocupar buenas posiciones en rankings como el Ad Age Ranking en EUA, el W \& V-Kreativ Ranking en Alemania o el The Big Won Report que antecede y predice los premios de Cannes.

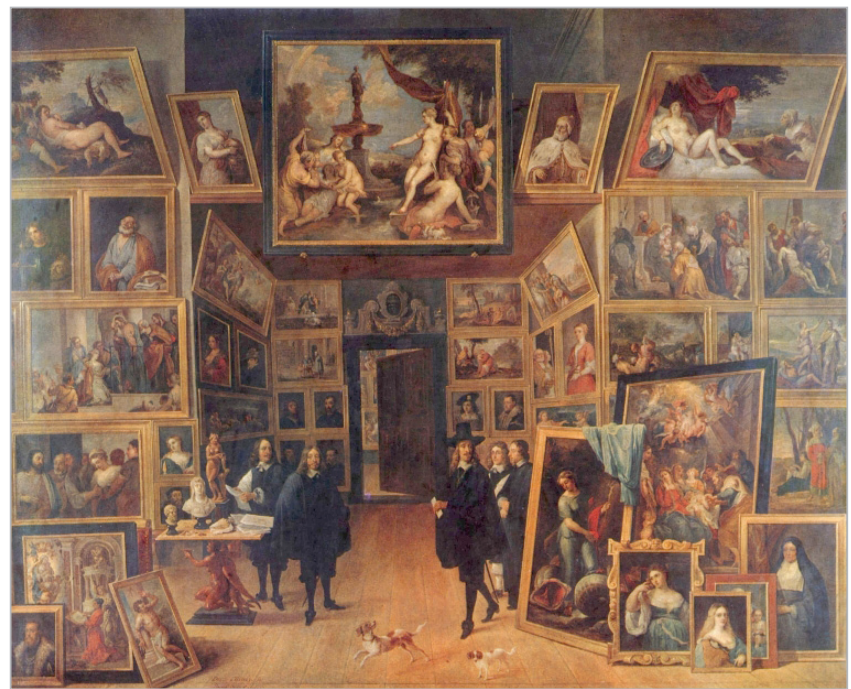

Figura 1. Metacuadro de David Teniers: El archiduque Leopoldo Guillermo en su galería de pinturas de Bruselas
En España los rankings de publicidad muestran una visión reducida y "marketiniana" del panorama publicitario, al medir aspectos concretos como la facturación en series temporales cortas. Ese es el caso del ranking de agencias de Infoadex, probablemente el más popular dentro del sector profesional, que establece un orden de agencias según la estimación del volumen de inversión gestionada en el último año (se calcula sobre precio-tarifa y no sobre precio real de las inserciones).

http://www.infoadex.es/estudios.html\#

En España los rankings de publicidad muestran una visión reducida y 'marketiniana' del panorama publicitario

Existen no obstante otros rankings en España que evalúan la creatividad y la excelencia, como:

- Premios control: clasificación que se elabora mensualmente durante un período global aproximado de 10 meses y según las valoraciones de un jurado de lectores que cambia cada mes y que pueden considerarse expertos si se tiene en cuenta que Control es una publicación especializada.

- Ranking de agencias españolas más premiadas de anuncios: ranking anual que determina el valor de cada agencia en función de los galardones que ha conseguido ese año; sin embargo, no estima diferenciación entre los distintos premios, lo que ha motivado críticas.

http://recursos.anuncios.com/files/470/54.jpg

En ambos casos se trata de meta-productos desarrollados por publicaciones especializadas en publicidad, que carecen de una base metodológica tan sólida y compleja como las de los rankings de publicidad de otros países. Esta ausencia justifica la creación de nuevas propuestas que superen los productos existentes y permitan analizar la industria publicitaria española.

Con este propósito se ha realizado el Ranking ESCO de agencias de publicidad que evalúa la calidad de las agencias a través de sus productos (los anuncios) utilizando un método indirecto (los premios que reciben) y según una ponderación compleja y sólida. En la actualidad las agencias publicitarias 
realizan diferentes trabajos que están poco o nada representados en los festivales de publicidad, como la llamada publicidad below the line. Es por ello que este ranking trata de aproximarse a la industria desde una sola dimensión, aunque muy significativa.

El objetivo de este trabajo es doble: por un lado reflexionar sobre el significado de un ranking basado en premios, y en segundo lugar realizar una breve exposición de las características del Ranking ESCO y sus resultados para la edición 2012. Los impulsores de este producto son ESCO (Escuela Superior de Comunicación y Marketing) de Granada y un conjunto de profesores de universidades españolas.

\section{El ranking, una lista ordenada}

En El vértigo de las listas, Umberto Eco (2009) reflexiona sobre cómo éstas han sido herramientas usadas para enumerar y describir los elementos de un sistema, desde la sociedad griega registrada en el listado de elementos que conformaban la descripción que Homero hacía del escudo de Aquiles hasta las listas normalizadas y jerárquicas elaboradas en el siglo XIX y que respondían a las sistematizaciones propias de la ciencia. Pero cuando una de las características del sistema es la competencia entre sus elementos, como en el caso de las agencias de publicidad, las listas se convierten en rankings.

Los premios conforman un parámetro sólido conforme al cual crear un ranking

Un ranking es una clasificación ordenada de mayor a menor de un conjunto de elementos de un sistema, de tal forma que su orden y posición relativa indican que los elementos que aparecen en primer lugar son, en la dimensión/es estudiada/s, más destacados que aquellos que aparecen con posterioridad. Los rankings normalmente miden parámetros directos; en el caso de los de agencias de publicidad, podemos decir parafraseando a Webster (1986) que deben ser realizados según un criterio o un conjunto de criterios que permitan medir o reflejar la calidad de las agencias.

\section{Festivales de publicidad}

El Ranking ESCO de agencias de publicidad se "alimenta" de los premios que las agencias reciben en los festivales de Publicidad. Helgesen (1994) indica que los objetivos de estos premios son dos:

- reconocimiento a una labor profesional concreta;

- ser un espacio publicitario para las agencias.

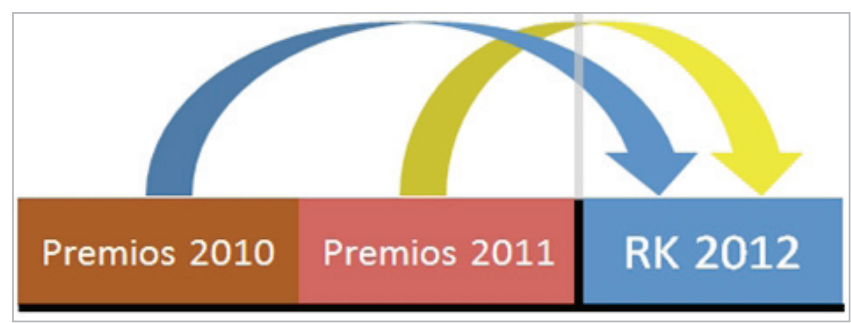

Figura 2. Diagrama de selección del período evaluado para el Ranking 2012

\begin{tabular}{|l|c|l|}
\hline \multicolumn{1}{|c|}{ Categoría } & Valor & \multicolumn{1}{c|}{ Festival } \\
\hline Nacional & 1 & Premios Ampe \\
\hline Nacional & 1 & Premios Aspid \\
\hline Nacional & 1 & Premios Inspirational \\
\hline Nacional & 1 & Publifestival \\
\hline Nacional + & 1,5 & Premios Chupete \\
\hline Nacional + & 1,5 & Premios Eficacia \\
\hline Nacional + & 1,5 & Premios Fiap \\
\hline Nacional + & 1,5 & Premios Ondas \\
\hline Internacional & 2 & El Sol \\
\hline Internacional & 2 & European Excellence Awards \\
\hline Internacional + & 2,5 & Cannes Lions \\
\hline Internacional + & 2,5 & Clio Awards \\
\hline Internacional + & 2,5 & Echo Awards \\
\hline
\end{tabular}

Tabla 1. Festivales utilizados en el ranking: categorización y ponderación

Los certámenes profesionales también son una forma de reconocer la calidad del trabajo publicitario. En publicidad, el aporte más significativo de los festivales es el prestigio y estatus (Helgesen, 1994; Schweitzer; Hester, 1992), puesto que los premios son meramente figurativos, si bien el prestigio implica otro tipo de reportes más significativos. Tippins y Kunkel (2006) han analizado las vinculaciones entre la obtención de un premio publicitario y la rentabilidad para la marca anunciante a corto plazo. No obstante, como establece Helgesen (1994), es difícil interpretar la relación entre los premios y los resultados de marketing, ya que éstos dependen también de otros factores ajenos a la publicidad. Este autor interpreta los premios como un indicador del rendimiento publicitario que reconoce la excelencia creativa de los productos publicitarios y sirve como guía para orientar prácticas eficaces (Helgesen, 1994). Percibidos como instrumentos de promoción para las agencias de publicidad, los premios son símbolos de liderazgo profesional que les permiten conseguir nuevos clientes y refuerzan las carreras personales de los publicistas (Helgesen, 1994).

Por todo ello y por su dinámica sujeta a unas normas de participación y a la evaluación de un jurado experto, los premios conforman un parámetro sólido conforme al cual crear un ranking. Otras posibilidades serían evaluar la eficacia, los ingresos atribuibles o el estudio de las estrategias de publicidad. Realizar un ranking en relación con estas variables supone un esfuerzo extraordinario si se pretende hacer a nivel nacional, en cambio la carga de datos de los premios es relativamente sencilla.

\section{Metodología del Ranking ESCO de agencias de publicidad}

El Ranking ESCO es un meta-producto que utiliza los premios que reciben los anuncios publicitarios en festivales (que a su vez son sistemas jerárquicos) para generar un listado ordenado de agencias, anuncios y anunciantes. Es además una ventana por la que se pueden apreciar el conjunto de los festivales que lo componen, como si fuese uno de los famosos "metacuadros" de Teniers (ver figura 1). En el Ranking ESCO se puntúan los anuncios ponderando los premios 
que reciben y los certámenes en los que son galardonados. Los anuncios que se recopilan son los realizados por agencias españolas y premiados en el extranjero, o los premiados en festivales españoles y que se han emitido en España sin importar la nacionalidad de la agencia productora. Por tanto es un ranking que mide a las agencias de publicidad españolas y a las extranjeras que compiten con ellas en el mercado nacional.

\section{Selección y ponderación de los festivales}

Existen múltiples festivales de publicidad, pero no todos tienen el mismo prestigio profesional y consecuentemente el mismo valor. Podrían diferenciarse estas categorías:

- nacional e internacional;

- especializados o generalistas;

- centrados en la eficacia o en la creatividad (Rosier, 2006), si bien Montano indica que "mayoritariamente los festivales publicitarios premian la creatividad mientras que la efectividad es valorada en un reducido número de premios" (Montano, 2004).

De forma general parece existir una relación directa entre la calidad de un festival y la calidad y número de sus concursantes. Siguiendo con la misma línea de razonamiento lógico, en la cantidad y diversidad también existen más posibilidades de calidad, motivo por el que los festivales de publicidad nacional tienen menos valor que los internacionales, ya que los últimos tienen en potencia más competidores.

Se han creado 4 grupos (tabla 1). Dentro de los nacionales, la diferenciación de las subcategorías responde a los resultados de un cuestionario de valoración que se realizó a los asesores y a otros especialistas del área ajenos al proyecto. Dicha consulta indicó la existencia de festivales nacionales mejor valorados (Nacional +) que otros. En la categoría internacional se definieron dos grupos: Internacional + , para los festivales de publicidad mundiales, e Internacional, para los internacionales por regiones (europeos y latinoamericanos). Si bien, para futuras ediciones del ranking, los asesores (expertos que participan en la realización del producto) revisarán y actualizarán la pertenencia de los festivales a la categoría correspondiente.

\section{Ponderación de los premios}

Evaluar los premios de una agencia significa indirectamente evaluar la calidad de la misma. Es una medida indirecta, puesto que es una valoración que realizan otros, principalmente especialistas consumados en el área, sobre la calidad de un producto publicitario. En los festivales se eligen los anuncios dignos de mención entre muchos aspirantes, de manera que en muchos casos se distinguen entre los ganadores, categorías de premios con diferente valor (oro, plata, mención de honor, etc.). No todos los festivales poseen idénticas categorías de premios, por lo que resulta necesario normalizar estos valores.

\section{El Ranking ESCO utiliza los premios que reciben los anuncios publicitarios en fes- tivales para generar un listado ordenado de agencias, anuncios y anunciantes}

La heterogeneidad de los festivales y de las categorías los hace difíciles de comparar. Su homogeneización siempre será convencional y tendrá un punto de arbitrariedad. Para la construcción del Ranking ESCO se han utilizado certámenes que tienen diversas casuísticas y terminologías de premios, desde los que no distinguen la calidad de los trabajos premiados (European Excellence Awards y Publifestival en 2011) hasta los que catalogan 6 tipos de premios según su valor (Echo Awards). Debido a ello, para procesar los diferentes premios hay que crear una tabla de equivalencias (tabla 2). En la ponderación se le ha dado valor 1 al premio de mayor categoría (Oro, Gran Premio, etc.), y conforme baja la categoría se les ha ponderado con valor 2, 3, 4 y 5 (estos valores se utilizan en el denominador de la ecuación, por lo tanto conforme mayores son, menor es la puntuación

\begin{tabular}{|c|c|c|c|c|c|c|}
\hline Festivales & 0,5 & 1 & 2 & 3 & 4 & 5 \\
\hline Cannes Lions & & Oro & Plata & Bronce & & \\
\hline Clio & & Oro & Plata & Bronce & Shortlist & \\
\hline Echo Awards & Platino & Oro & Plata & Bronce & Leaders & Finalista \\
\hline El Sol & Platino & Gran premio & Oro & Plata & Bronce & \\
\hline $\begin{array}{l}\text { European Excellence } \\
\text { Awards }\end{array}$ & & \multicolumn{2}{|c|}{ Sin distinción } & & & \\
\hline Premios Ampe & & Oro & Plata & & & \\
\hline Premios ASPID & Platino & Mejor & Oro & Plata & & \\
\hline Premios Chupete & & Gran premio & Premio especial & \multicolumn{2}{|c|}{ Sin distinción } & \\
\hline Premios Eficacia & & Gran premio & Oro & Plata & Bronce & \\
\hline Premios Fiap & $\begin{array}{c}\text { Gran premio/ } \\
\text { Platino }\end{array}$ & Oro & Plata & Bronce & Finalista & \\
\hline Premios Inspirational & Gran premio & Oro & Plata & Bronce & & \\
\hline Premios Ondas & Mejor & \multicolumn{2}{|c|}{ Sin distinción } & & & \\
\hline Publifestifal & & $\begin{array}{l}\text { Primer } \\
\text { premio }\end{array}$ & Segundo premio & $\begin{array}{c}\text { Tercer } \\
\text { premio }\end{array}$ & \multicolumn{2}{|c|}{ Cuarto premio } \\
\hline & & \multicolumn{5}{|c|}{ En 2011 los premios no se distinguen } \\
\hline
\end{tabular}

resultante). En algunos festivales se premia de manera extraordinaria algún anuncio (nunca más de tres) con galardones que reconocen en la mayoría de los casos el mejor anuncio de todas las categorías para ese año (como el premio de Platino del festival El Sol); a tales premios se les ha otorgado excepcionalmente valor 0,5 . Por otro lado, los premios de certámenes que no establecen distinciones entre los mismos se han ponderado con valor 1 . 


\section{Cálculo del valor de las agencias}

Para la selección de los años de la carga se ha utilizado algo similar al journal impact factor de Garfield (2006). Así, para calcular el valor de una agencia en un año concreto se tendrán en cuenta los premios recibidos en los dos años previos (figura 2), con lo que se obtiene un valor muy aproximado de la situación actual. Una ventana de premios superior a dos años generaría un ranking con posiciones (especialmente las importantes) poco móviles, de tal forma que cada edición apenas aportaría información sobre la anterior. Con el sistema propuesto, en cada edición se incorporan el $50 \%$ de los datos, por lo que existe un factor de continuidad y otro de novedad igualados.

Evaluar los premios de una agencia significa indirectamente evaluar la calidad de la agencia

Una vez ponderados los festivales según categorías (tabla 1) y los premios recibidos en los mismos (tabla 2), se procede al cálculo de los valores del ranking. Para ello se suman los puntos obtenidos por cada anuncio y se normalizan utilizando raíces cuadradas para atemperar la diferencia entre elementos, como hace el Ranking de Shangai (Docampo, 2013). Este cálculo se hace cambiando el nivel de agregación para los diferentes rankings (anuncios, agencias y anunciantes), de tal forma que se le da valor 10.000 a:

a) El anuncio que mayor número de premios ha recibido (ranking de anuncios);

b) La agencia de publicidad que acapara mayor número de puntos sumando los valores de sus anuncios (ranking de agencias);

c) La empresa anunciante cuyas piezas han recibido el mayor número de puntos (ranking de anunciantes).

El valor de las agencias de publicidad y las empresas anunciantes surge, en la metodología de este ranking, de la suma de sus anuncios, normalizado respecto a aquel que ocupa la primera posición. La fórmula 1 muestra el cálculo utilizado para medir el valor de una agencia en el ranking.

$$
\text { Agencia }_{z}=\frac{\sum_{i=1}^{m} \sum_{j=1}^{n} \frac{F_{i}}{A n_{j}}+\sum_{x=1}^{p} \sum_{y=1}^{q} \frac{F_{x}}{A n_{y}}}{\left(\text { Agencia }_{\alpha}\right)^{1 / 2}}
$$

Fórmula 1. Fórmula del ranking de agencias

Valor normalizado de la agencia " $\mathrm{z}$ ":

z: agencia evaluada

$\mathrm{m}$ : festival

$\mathrm{n}$ : posición del anuncio dentro del festival

Fi: valor del festival en 2010

$A n_{j}$ : valoración del premio recibido en el festival en 2010

$\mathrm{p}$ : festival

q: posición del anuncio dentro del festival

$\mathrm{F}_{\mathrm{x}}$ : valor del festival en 2011

$A n_{\mathrm{y}}$ : valoración del premio recibido en el festival en 2011

Para comprender mejor la fórmula hay que tener en cuenta que:

- un anuncio producido por dos o más agencias no reparte su valor entre ellas, sino que puntúa como un anuncio único para cada una;

- un anuncio puede recibir más de un premio en un festival, éstos se acumulan en el valor definitivo sumándose.

\section{Resultados}

En la figura 3 se muestra la interfaz del Ranking ESCO de agencias de publicidad, así como el top ten de agencias para 2012. Un aspecto significativo del ranking es su transparencia: al pinchar sobre cada elemento (agencias, anuncios y anunciantes), la web ofrece la información que hay detrás del cálculo de la puntuación del mismo, lo que permite reproducir el sistema y a la vez otorga al usuario la capacidad de comprender cómo se calcula. Además cuenta con una ventaja adicional: los errores son fácilmente localizables y rectificables.

\section{Ranking}

\section{Tabla: RK. AGENCIAS}

\begin{tabular}{|l|}
\hline RK. Agencias \\
\hline RK. Anuncios \\
\hline RK. Anunciantes \\
\hline \\
\hline Equipo \\
\hline Método \\
\hline Publicaciones \\
\hline Festivales \\
\hline Ayuda \\
\hline
\end{tabular}

Buscar

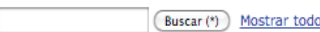

OFrase exacta O Todas las palabras e Cualauier palabra

\begin{tabular}{|c|c|c|c|}
\hline POSICTÓNA & MOMBRE (*) & PUNTUACrón & \\
\hline 1 & MCCANN ERICKSON & 10000 & 狊 \\
\hline 2 & SHACKLETON & 9400 & 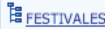 \\
\hline 3 & SRA. RUSHMORE & 7027 & 畺FESTIVA \\
\hline 4 & BASSAT OGILVY & 6985 & LESTIVA \\
\hline 5 & LEO BURNETT IBERIA & 6582 & LEESTIVA \\
\hline 6 & DDB & 6066 & RegETIVA \\
\hline 7 & JWT & 5979 & EESTIVA \\
\hline 8 & TBWA & 5449 & IIESTIVA \\
\hline 9 & GERMINAL COMUNICACIÓN & 5245 & UEESTIVA \\
\hline 10 & SAATCHI \& SAATCHI & 4801 & 品FESTIVA \\
\hline
\end{tabular}

Figura 3. Interfaz del Ranking ESCO de agencias de publicidad http://ranking.escogranada.com
Esta transparencia permite al usuario averiguar datos importantes de los diferentes objetos estudiados, como las agencias creadoras, las marcas que hay detrás, los premios que ha recibido cada anuncio (festival, año y premio).

\section{Ranking de agencias, anuncios $y$ anunciantes}

Una vez normalizados y ponderados los datos, el ranking para 2012 registra 197 agencias, 611 anuncios y 359 anunciantes. En la tabla 4 se observa el top ten 
de cada uno de los objetos estudiados. La agencia con mayor puntuación es McCann Erickson, que presenta 53 anuncios cuya suma de premios asciende a 103 en 9 festivales diferentes. De la misma forma el anuncio que encabeza el ranking de anuncios es "Pastillas contra el dolor ajeno" de la agencia Germinal Comunicación que ha recibido 11 premios en 5 festivales. Coca Cola encabeza el ranking de anunciantes, con 18 anuncios que han recibido un total de 42 premios diferentes. Además el Ranking nos permite ver con qué agencias ha trabajado Coca Cola en el período 20102011 (Grey, McCann Erickson y Sra. Rushmore).

Otro aspecto llamativo de los resultados es la existencia de élites de agencias. De las 196 recopiladas, sólo 9 poseen valores superiores a 5.000 puntos, lo que indica que éstas y sus anuncios han acaparado una gran parte de los premios de los festivales nacionales analizados y son las principales agencias españolas que han conseguido premios en certámenes internacionales.

\section{Consideraciones finales}

Se ha presentado el Ranking ESCO de agencias de publicidad, poniéndolo en contexto y explicando su metodología. Se hace necesario subrayar para su correcta interpretación, que este ranking se limita a aquellas empresas que participan en los festivales y premios reseñados en la tabla 1 , siempre y cuando sean: a) españolas o b) extranjeras que trabajan en España (sólo se valorarán sus trabajos en España). Por tanto las compañías de publicidad que no participan y reciben premios en los festivales están fuera del producto. Probablemente este aspecto suponga uno de los elementos de crítica a la metodología del ranking. Los motivos por los que las agencias no participan en certámenes son diversos, a pesar de los beneficios aparentes que suponen. Chipperfield (1989) citado en Polonsky y Waller (1995), indicaba que la razón de fondo por la que muchas agencias criticaban

\begin{tabular}{|l|l|}
\hline \multirow{2}{*}{ Agencias } & $\begin{array}{l}\text { Anuncios premiados (festival, año, marca y categoría } \\
\text { del premio) }\end{array}$ \\
\hline \multirow{2}{*}{ Anuncios } & Agencia creadora \\
\cline { 2 - 2 } & Marca \\
\cline { 2 - 2 } Anunciantes & $\begin{array}{l}\text { Premios recibidos (festival, año y premio) } \\
\text { cidad y categoría del premio) }\end{array}$ \\
\hline
\end{tabular}

Tabla 3. Datos facilitados por el Ranking

este tipo de premios se debe a que sus campañas publicitarias son mediocres en comparación con el resto, lo que no es óbice para que obtengan pingües beneficios.

En los resultados se aprecia que las agencias mejor situadas son internacionales. En el ranking de anunciantes llama la atención la inclusión de ONGs (Médicos Sin Fronteras y Amnistía Internacional) en puestos de relevancia. En el de anuncios lo más destacable es que la pieza más premiada, Pastillas contra el dolor ajeno (Médicos Sin Fronteras), está creada por una agencia nacional (Germinal Comunicación).

\section{Existe una relación directa entre la cali- dad de un festival y la calidad y número de sus concursantes}

Creemos que además de servir para ordenar las agencias y anuncios por su calidad, el ranking es un buen espejo donde estudiar la publicidad española.

En futuras ediciones el ranking se enriquecerá con nuevos apartados, como directorios de agencias, donde se podrá consultar la dirección de las mismas, así como una muestra de los anuncios más premiados. Asimismo se pretende mejorar la calidad del producto incluyendo nuevos festivales.

\begin{tabular}{|c|c|c|c|c|c|c|}
\hline \multirow{2}{*}{ Posición } & \multicolumn{2}{|c|}{ Ranking de agencias } & \multicolumn{2}{|l|}{ Ranking de anuncios } & \multicolumn{2}{|c|}{ Ranking de anunciantes } \\
\hline & Nombre & Puntos & Nombre & Puntos & Nombre & Puntos \\
\hline 1 & McCann Erickson & 10.000 & Pastillas contra el dolor ajeno & 10.000 & Coca Cola España & 10.000 \\
\hline 2 & Shackleton & 9.400 & Capacitados & 8.043 & Sony & 7.357 \\
\hline 3 & Sra. Rushmore & 7.027 & Hotel Corona Save The beach & 7.276 & Médicos Sin Fronteras & 7.235 \\
\hline 4 & Bassat Ogilvy & 6.985 & Reponte y camina & 7.276 & Campofrío & 7.134 \\
\hline 5 & Leo Burnett Iberia & 6.582 & Misión di no al Winter Blues & 6.642 & Gmodelo & 6.519 \\
\hline 6 & $D D B$ & 6.066 & El primer vídeo de YouTube que cuesta 1,20€ & 6.340 & ING Direct & 6.130 \\
\hline 7 & $J W T$ & 5.979 & Cambiazo & 5.622 & Movistar & 6.020 \\
\hline 8 & TBWA & 5.449 & Cuatro sentidos & 5.445 & Volkswagen Audi España & 5.783 \\
\hline 9 & Germinal Comunicación & 5.245 & Internet móvil & 5.144 & Amnistía Internacional & 5.434 \\
\hline 10 & Saatchi \& Saatchi & 4.801 & Quique the head & 5.144 & Reckitt Benckisser & 5.309 \\
\hline 11 & Contrapunto BBDO & 4.714 & Shackleton & 5.144 & Promotur & 5.245 \\
\hline 12 & Zapping/M\&CSaatchi & 4.664 & La cocina de calle 13 & 5.048 & Ayuntamiento de Pamplona & 4.846 \\
\hline 13 & Euro RSCG & 4.331 & Cardeñosa & 5.000 & FIAT & 4.776 \\
\hline 14 & Publicis & 4.276 & Encuentro & 5.000 & FSC Inserta & 4.487 \\
\hline 15 & Remo & 4.200 & Víctor & 4.696 & Renfe & 4.487 \\
\hline 16 & HC Barcelona & 3.818 & Nombres & 4.537 & Telefónica & 4.487 \\
\hline 17 & Doubleyou & 3.787 & Fear & 4.428 & Spanair & 4.474 \\
\hline 18 & CP Proximity & 3.685 & Gifts & 4.428 & Mercedes Benz & 4.386 \\
\hline 19 & GAP'S & 3.563 & Pain & 4.428 & Unicef & 4.309 \\
\hline 20 & ${ }^{*} S, C, P, F$ & 3.547 & The Swap & 4.428 & Renault & 4.256 \\
\hline
\end{tabular}

Tabla 4. Top ten de los rankings de agencias, anuncios y anunciantes 


\section{Agradecimientos}

Este trabajo no habría sido posible sin la colaboración desinteresada de los asesores del proyecto, ni la dedicación de los técnicos que han diseñado la web. También queremos destacar el trabajo de Miguel-Ángel Rodríguez-Pinto como coautor del proyecto.

http://ranking.escogranada.com/equipo.html

\section{Bibliografía}

Chipperfield, Mark (1989). "Awards or sales". Marketing, v. February.

Docampo, Domingo (2013). "Reproducibility of the Shanghai academic ranking of world universities results". Scientometrics, v. 94, n. 2, pp. 567-587. http://dx.doi.org/10.1007/s11192-012-0801-y

Eco, Umberto (2009). El vértigo de las listas. Barcelona: Lumen. ISBN: 9788426417435

Garfield, Eugene (2006). "The history and meaning of the Journal impact factor". JAMA, v. 295, n. 1, pp. 90-94 http://garfield.library.upenn.edu/papers/jamajif2006.pdf

Helgesen, Thorolf (1994). "Advertising awards". Journal of advertising research, August, pp. 43-54

Kübler, Raoul V.; Proppe, Dennis (2012). "Faking or convincing: why do some advertising campaigns win creativity awards?" BUR: Business research, v. 5, n. 1, pp. 60-81. http://papers.ssrn.com/sol3/papers.cfm?abstract $i d=2064644$

Montano, Jesse-Raúl (2004). "Characteristics of U.S. Hispanic advertising: a comparison of awar-winning and nonaward winning commercials". University of Florida. http://etd.fcla.edu/UF/UFE0005381/montano_j.pdf

Polonsky, Michael J.; Waller, David S. (1995). “Does winning advertising award pay? The Australian experience". Journal of advertising research, v. 35, n. 1

http://www.accessmylibrary.com/article-1G1-76914148/ does-winning-advertising-awards.html

Rosier, Scarlett Whitney (2006). "Comparison of U.S. and Latin American Clio award winning advertisements". University of Florida.

http://ufdcimages.uflib.ufl.edu/UF/E0/01/62/02/00001/ rosier_s.pdf

Schweitzer, John C.; Hester, Joe B. (1992). "The importance of winning advertising award shows". Southwestern mass communication, v. 7, n. 1, pp. 55-66.

Tippins, Michael J.; Kunkel, Robert A. (2006). "Winning a Clio advertising award and its relationship to firm profitability". Journal of marketing communications, v. 12, n. 1, pp. 1-14.

http://dx.doi.org/10.1080/13527260500247942

Webster, David S. (1986). Academic quality rankings of American colleges and universities. Springfield: Charles C. Thomas. ISBN: 9780398052126

\section{El Directorio EXIT permite localizar colegas para participar en cursos, conferencias, proyectos, o recibir ofertas de trabajo. \\ ¿Te apuntas? \\ Ya somos 2.900 profesionales de la información}

Para bibliotecarios, documentalistas, periodistas, comunicadores, informáticos y diseñadores que ya figuren al menos en Google Scholar, o tengan experiencia ejerciendo en empresas o instituciones. http://directorioexit.info

\section{exit}

Nieves González-Fernández-Villavicencio •


Y acabamos de estrenar nuevo look y nuevas funciones... 